\title{
Correspondence
}

\section{Ionisers in the management of bronchial asthma}

SIR,-Drs SG Nogrady and SB Furnass have reported a well planned and carefully controlled trial (December 1983, p 919). However, their negative conclusions should not be taken at their face value.

An examination of their table 2 reveals much of interest. If the results for each of the four assessments are ranked from 1 to 5 according to how the columns compare with one another, we get the table below.

Results set out in the ranking of results for the four assessments ( 1 = most favourable, $5=$ least favourable)

\begin{tabular}{|c|c|c|c|}
\hline \multirow{2}{*}{$\begin{array}{l}\text { Group } 1 \\
\text { Active } \\
\text { generator }\end{array}$} & \multirow[b]{2}{*}{$\begin{array}{l}\text { No } \\
\text { generator }\end{array}$} & \multirow{2}{*}{$\begin{array}{l}\text { Group } 2 \\
\text { Active } \\
\text { generator }\end{array}$} & \multirow[b]{2}{*}{$\begin{array}{l}\text { No } \\
\text { generator }\end{array}$} \\
\hline & & & \\
\hline $\begin{array}{l}4 \\
5 \\
3 \\
5\end{array}$ & $\begin{array}{l}1 \\
1 \\
1 \\
2\end{array}$ & $\begin{array}{l}1 \\
1 \\
1 \\
1\end{array}$ & $\begin{array}{l}2 \\
3^{1 / 2} \\
4 \\
2\end{array}$ \\
\hline
\end{tabular}

If group 2 were considered on its own the active generator effect would undoubtedly be significant $(p<0.01)$, and there is evidence also of some carry over effect into the next period. That there was a positive effect is further supported by the consistency of the average scores in the three periods before the generator was used. However, group 2 scores cannot be considered in isolation from group 1 . Inspection of group 1 results suggests a more pronounced lag effect, scores being at their best in the period immediately following the active generator for three out of four assessments and then gradually but consistently declining over the next two (placebo and untreated) periods.

The findings are therefore consistent with the hypothesis of a positive effect produced by negative ionisers in asthmatic patients, with a time lag evident for the appearance of benefit after the start of use, and a further time lag after cessation of use before the benefit disappears. One may add a speculation that the greater time lag before benefit in group 1 perhaps resulted from initial apprehensions or disappointments regarding the effects of treatment, which were no longer present by the time the active generator was introduced for group 2.

The apparent inconsistency of results in the published studies on the effects of negative air ions, many of which are quoted by the authors, is typical of a situation where the fundamental physiological mechanism has not been elucidated. Consequently we do not know the answers to two questions which would be essential for planning a definitive trial-namely, whether or not there is a time lag in effect; and whether the effect, if it exists, is likely to affect all subjects or only a proportion. Both these possibilities were discussed in an earlier paper in Thorax, ${ }^{\prime}$ as they have been in other work on the subject, and the sec- ond question has been dealt with extensively by Sulman. ${ }^{2}$ ?

Moreover, Drs Nogrady and Furnass have made no $\vec{F}$ mention in their statistical assessment of the possibility of a $\stackrel{9}{+}$ type 2 error-that is, of wrongly accepting the null $\bar{C}$ hypothesis. Such an error becomes very likely when the $\underline{\sigma}$ standard deviations of the parameters chosen are high in $\bar{\sigma}$ relation to the mean, as was the case with all of their four $\vec{\Phi}$ measures, and the power to detect a positive result is correspondingly low. For all these reasons therefore it is to be $\omega$ hoped that their paper will not be used to discourage $\vec{\circ}$ further research into this most interesting subject.

KEVIN BROWNE $\vec{\omega}$

66A Warwick Way London SW1

' Jones DP et al. Effect of long term ionised air treatment on
patients with bronchial asthma. Thorax 1976;31:428-32.
'Sulman FG et al. Absence of harmful effects of protracted nega-
tive air ionisation. Int $J$ Biometeor 1978;22:53-8.
** This letter was sent to the authors, and Dr Nogrady
*eplies below.

SIR,- - In our original assessment we too noticed that if the two groups were analysed separately there appeared to be $\overline{0}$ differences between the groups in their response to ionis-ڤ્ㅇ ers. Group 2 did show some benefits. In our original draft@ we considered this, but on reflection felt that it was not $\overrightarrow{\vec{O}}$ valid in a double blind crossover study to look at the윽 groups separately.

Our groups, however, were not very well balanced Indeed as group 2 contained more female subjects it would be possible to speculate that there were sex differences in $\vec{f}$ the response. The size of the group, however, does not $\frac{\mathrm{O}}{2}$ permit this. We too noticed that one could speculate that $\times$ differences in the time of onset of the effective ionisers and a lag phase where such benefits might slowly wash out could be used to seek a positive result. I do not believe, however, that this gets around the problem of lack of 3 response from the group 1 patients. It would be quite poss- ible to test hypotheses about age, sex, severity of asthma, and so on by using an appropriate factorial design with complete counterbalancing for each group. We think that such studies should be done.

We do not state that this study excludes any potential benefit from negative ion generators. What we are saying is $N$ that there are inadequate grounds to recommend their pre-Nు scription to patients suffering from asthma.

SG NOGRADY Respiratory Unitc

Royal Canberra Hospital Acton ACT 2601. Australia 